\title{
A practical compact source of heralded single photons for simple detection LIDAR
}

\author{
Mateusz P. Mrozowski, John Jeffers, and Jonathan D. Pritchard \\ Department of Physics, University of Strathclyde, John Anderson Building, \\ 107 Rottenrow East, Glasgow G4 0NG, United Kingdom
}

\begin{abstract}
Optical quantum technologies such as quantum sensing, quantum cryptography and quantum computation all utilize properties of non-classical light, such as precise photon-number and entangled photon-pair states, to surpass technologies based on the classical light. A common route for obtaining heralded single photons is spontaneous four-wave mixing in optical fibers, allowing for a well-defined spatial mode, for high efficiency integration into optical fiber networks. These fibers are typically pumped using large, commercial, pulsed lasers requiring high-power $(\sim 10 \mathrm{~W})$ pump lasers and are limited to $\sim \mathrm{MHz}$ repetition rate. Here we propose a costefficient, compact and mobile alternative. Photon pairs at $660 \mathrm{~nm}$ and $960 \mathrm{~nm}$ will be created using four-wave mixing in commercial birefringent optical fiber, pumped using transform limited picosecond pulses with $\mathrm{GHz}$ repetition rates derived from a $785 \mathrm{~nm} \mathrm{CW}$ laser diode using cavity-enhanced optical frequency comb generation. The pulses are predicted to have average power of $275 \mathrm{~mW}$, a peak power of $>40 \mathrm{~W}$, and predicted photon yield of $>2000$ pairs detected per second. This design will be later utilized to implement a quantum illumination scheme based on a coincidence count between idler and signal photons - instead of joint measurement between signal and idler. This will allow for quantum advantage over classic LIDAR without the requirement for maintaining an interferometric stability in free space.
\end{abstract}

Keywords: Quantum information, Quantum optics, Nonlinear optics, Fiber optics, Ultrafast optics

\section{INTRODUCTION}

Photon pairs are an essential resource, either as factorisable states of pure photons for applications such as quantum computation with linear optics,${ }^{1}$ and quantum simulations using photons, ${ }^{2}$ or as entangled pairs used to enhance timing measurements, ${ }^{3}$ improve clock synchronization,${ }^{4}$ cancel dispersion in interferometer $\$^{5}$ and for quantum illumination. 6 [10

Whilst entangled light sources enable quantum illumination to achieve a theoretical detection enhancement over classical illumination, ${ }^{7}$ developing a detection scheme able to utilise this regime in practice - without sensitivity to decoherence or need for an interferometric stability on a length scale of an optical wavelength, was so far possible only within an optical fiber 11 Recent proposals have shown that quantum illumination using heralded rather than entangled photons can also be exploited in LIDAR schemes $\frac{12}{13}$ in which simple detection based on coincidence measurements using single photon detector modules has been demonstrated to enable rejection of thermal background and robustness against spoofing. $]^{[7}$

Two common routes to obtain heralded photons are Spontaneous Parametric Down Conversion (SPDC) in non-linear crystals, $\frac{14}{14}$ and Spontaneous Four-Wave Mixing (SFWM) in birefringent optical fibers $\frac{15}{15}$ The latter offers advantages in the form of a well defined spatial mode for high efficiency coupling into optical fiber networks developed for quantum information protocols, $\frac{16}{18}$ as well as the lower material cost of optical fibers, compared to the cost of non-linear photonic crystals. Recent studies have shown that using SFWM in commercial grade polarisation-maintaining single-mode optical fibers provides a high purity and wavelength tuneable source of heralded single photons, adaptable to both visible ${ }^{[15}$ and telecoms wavelengths. ${ }^{[19}$

In typical experiments, photon pairs are obtained by pumping birefringent fibers using bulky, high power $(\sim 10 \mathrm{~W})$ pulsed laser systems, which come with significant price-tag $(\sim £ 100 \mathrm{k})$, and a fixed repetition rate

M.M.: E-mail: mateusz.mrozowski@strath.ac.uk 
$(\sim 1-100 \mathrm{MHz})$. Here we propose an alternative low cost and compact, heralded photon source, offering a high repetition rate $(\sim \mathrm{GHz})$, and a tuneable pulse width $(1-10 \mathrm{ps})$ that will allow for temporal control of the generated photons. This source uses an optical frequency comb generator (OFCG) based on an intra-cavity electro-optical modulator (EOM) to convert a CW pump into a mode-locked pulse train.20 22 A dual-cavity design is adopted to achieve a high efficiency output, 23 overcoming the major limitation of a single cavity OFCG sources. We estimate a photon pair detection rate of $>2000$ photon pairs/s following simulation of our pump source, currently limited by the damage threshold of the intra-cavity EOM.

\section{OPTICAL FREQUENCY COMB GENERATION}

\subsection{Single cavity OFCG}

A simple OFCG source involves use of an EOM inside an optical cavity driven resonantly with the cavity free spectral range (FSR) to generate a comb of phase-coherent sidebands that convert a CW pump laser into a train of short optical pulses with a repetition rate equal to twice the modulation frequency. The output electric field can be calculated by modelling the round-trip propagation as illustrated in Fig. 1(a) using the mathematical model presented in Sec. A.1, resulting in an output field $\vec{E}_{\text {out }}$ equal to

$$
\vec{E}_{\text {out }}=t_{\mathrm{c}} \hat{\phi}\left(\frac{\phi_{k}}{2}\right) \times M(\delta) \times t_{2}\left(\left[\mathbb{1}-r_{1} r_{2} t_{\mathrm{c}}^{2} \hat{\phi}\left(\phi_{k}\right) \times M(2 \delta)\right]^{-1} \times t_{1} \vec{E}_{\mathrm{in}}\right)
$$

where $\hat{\phi}\left(\phi_{k}\right)$ and $M(2 \delta)$ are the full round trip phase change operator and full round trip coupling matrix respectively, $\delta$ is the modulation depth in radians, $\phi_{k}$ is the round-trip phase change of $k^{\text {th }}$ sideband, $t_{i}$ and $r_{i}$ are transmittance and reflectivity of the mirror $M_{i}, t_{\mathrm{c}}$ is transmission through the EOM crystal, and $\vec{E}_{\mathrm{in}}$ is the single frequency optical field represented as a vector with central mode $(k=0)$ having an intensity equal to 1. This provides the electric field represented in the frequency domain from which the temporal response can be obtained using a Fourier transform. The frequency spectrum of the output exhibits an exponential decay in the

(a)
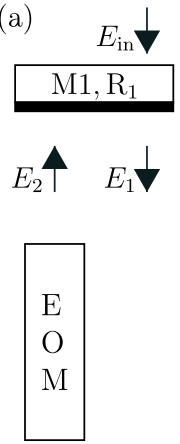

$\mathrm{M} 2, \mathrm{R}_{2}$

$E_{\text {out }}$

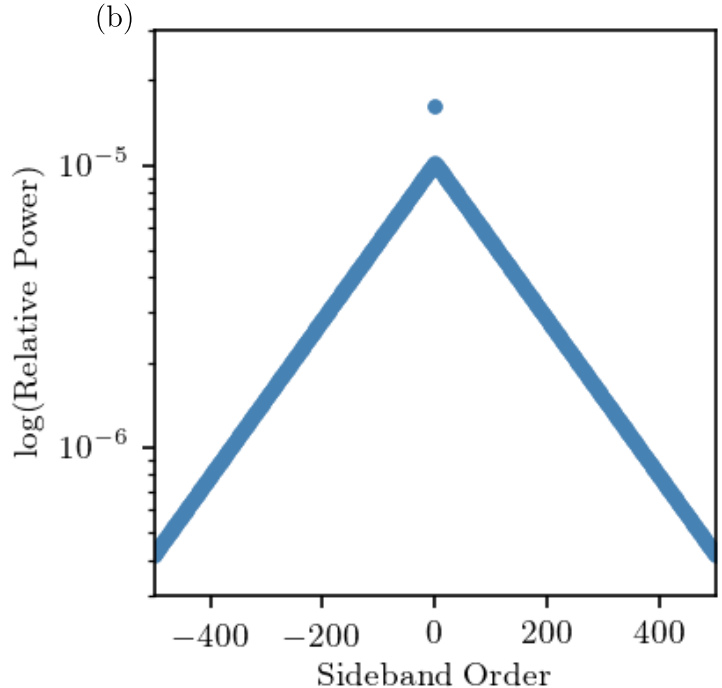

Sideband Order

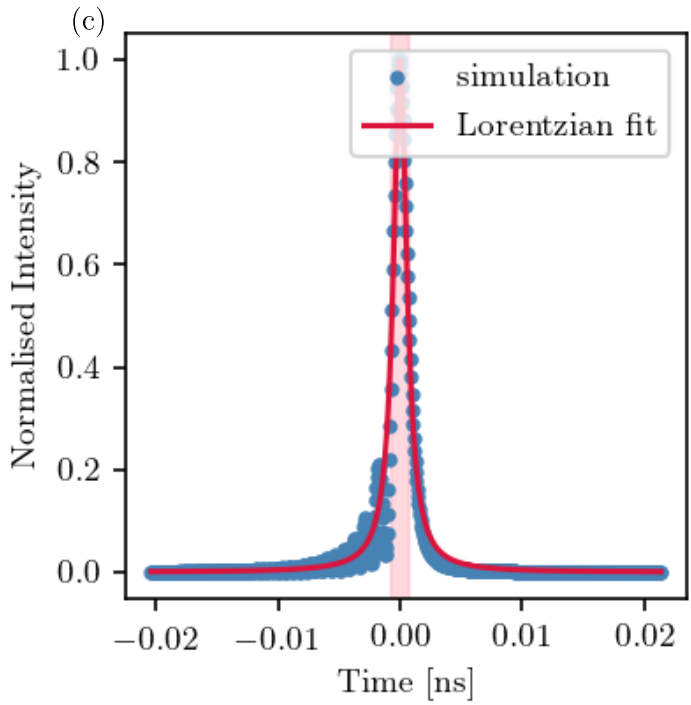

Figure 1: (a) Model of single cavity OFCG. Here we are using an approximation, in which light passes through the EOM only once per round trip, while the crystal is assumed to be twice as long and modulation depth is doubled. (b) Spectral output of the single cavity OFCG. Efficiency of the system $\eta=0.3 \%$. (c) Temporal output of the single cavity OFCG with a FWHM pulse $\Delta \tau=1.3 \mathrm{ps}$ as indicated by the red shaded area. The asymmetry is an artifact of the crystal dispersion. 
electric field as a function of sideband order, ${ }^{21}$ therefore the pulse shape will be Lorentzian of the form ${ }^{22}$

$$
E(t) \propto\left[\frac{1}{1+\left(4 f_{m} \delta F t\right)^{2}}\right] E_{\mathrm{in}},
$$

where $f_{m}$ is the modulation frequency, and $F$ is the cavity finesse. The pulse width $\Delta \tau_{p}$ at FWHM intensity can then be approximated as 22

$$
\Delta \tau_{p} \approx \frac{\sqrt{\sqrt{2}-1}}{2 f_{m} \delta F} .
$$

In the system consisting of an EOM placed in the cavity, the dispersion comes from both the dispersive effect of the electro-optic crystal, as well as the dispersion of the cavity mirrors. The latter can be neglected because it is small compared to the dispersive effect of the crystal. The full round trip phase change per sideband is given by 24

$$
\phi_{k}=\frac{k^{2}}{2} \operatorname{GVD}\left(2 \pi f_{m}\right)^{2} L_{c},
$$

where $k$ represents the sideband order, $f_{m}$ is the frequency with which the EOM is modulated, GVD is the group velocity dispersion of the material, and $L_{c}$ is the crystal's length. The phase difference between the carrier and the sidebands generated by the EOM increases quadratically with sideband order. Eventually that difference in phase will be large enough for the sideband to no longer be resonant with the optical cavity, because of the destructive interference that light mode will be suppressed, and in effect the frequency comb will be truncated. This dispersion limit produces a sharp cut-off in the optical frequency comb spectrum, limiting it's span $t \mathrm{c}^{24}$

$$
\Delta f=\frac{[2(-\beta+\delta) G]^{1 / 2}}{\pi},
$$

where $\beta$ is the normalized detuning between the input laser frequency $f_{o}$ and the nearest cavity resonance $f_{r}$, defined as $\beta=\left[\pi\left(f_{o}-f_{r}\right)\right] /$ FSR, $\delta$ is modulation depth, and $G$ is related to the EOM material dispersion by $G=2 \pi c / D \lambda_{o}^{2} L_{c}$, where $L_{c}$ is the crystal length, $\lambda_{o}$ is the input laser wavelength in vacuum and $D=\left.\frac{\lambda_{o}}{c} \frac{\partial^{2} n}{\partial \lambda^{2}}\right|_{\lambda=\lambda_{o}}$ is the material dispersion which can be related to the group velocity dispersion by use of ${ }^{201} \mathrm{GVD} \cong D \lambda_{o}^{2} / 2 \pi c$.

Using this model we extract typical performance for a single cavity system using the following parameters. Mirrors 1 and 2 were set to equal reflectivity $R_{1}=R_{2}=0.99$, with the EOM modulation depth $\delta=\pi / 2$, and $f_{m}=2.39 \mathrm{GHz}$. Due to the light passing twice through the EOM, it experiences a modulation of $2 \delta$ meaning for $\delta>\pi / 2$ the light becomes resonant with the next cavity mode leading to an unstable output rate and uneven intervals between output pulses 20,25

The EOM is modelled as an $L_{c}=20 \mathrm{~mm}$ magnesium-doped lithium tantalate crystal (MG:LiTaO $\left.{ }_{3}\right)$ chosen for its linear group velocity dispersion $\left(\mathrm{GVD}=308 \mathrm{fs}^{2} / \mathrm{mm}^{26}\right)$ at the central wavelength of $\lambda_{0}=785 \mathrm{~nm}$ and assumed to have an anti-reflective (AR) coating with $R=0.01 \%$ on each facet. The output electric field generated in the single cavity can be seen in Fig. 1.(b). As expected, in the frequency domain we can observe an exponential relationship between sidebands leading to a Lorentzian temporal output pulse, as shown in Fig. 11(c). The resulting FWHM pulse width $\Delta \tau_{p}=1.3 \mathrm{ps}$ and the efficiency of the system $\eta=0.3 \%$ at a repetition rate of $2 f_{m}=4.78 \mathrm{GHz}$.

\subsection{A Coupled Cavity OFCG}

A major limitation of the single cavity OFCG is the trade-off between increasing the modulation depth and the cavity finesse to achieve shorter pulses, and the increased loss from the central cavity mode resulting in an impedance mis-match that inhibits efficient mode-matching into the cavity and hence a low output power ${ }^{20}$ For the parameters presented in previous section the efficiency of the device was predicted to be only $\eta=0.3 \%$, with $<1 \%$ typical for experiments 2027

This limitation can be overcome using a coupled cavity configuration as demonstrated in 23 and shown schematically in Fig. 2(a). Here a planar mirror is placed between the input mirror M1 and the EOM crystal surface to split the OFCG into two separate cavities - an empty coupling cavity (M1 + M2), and a second cavity (M2 

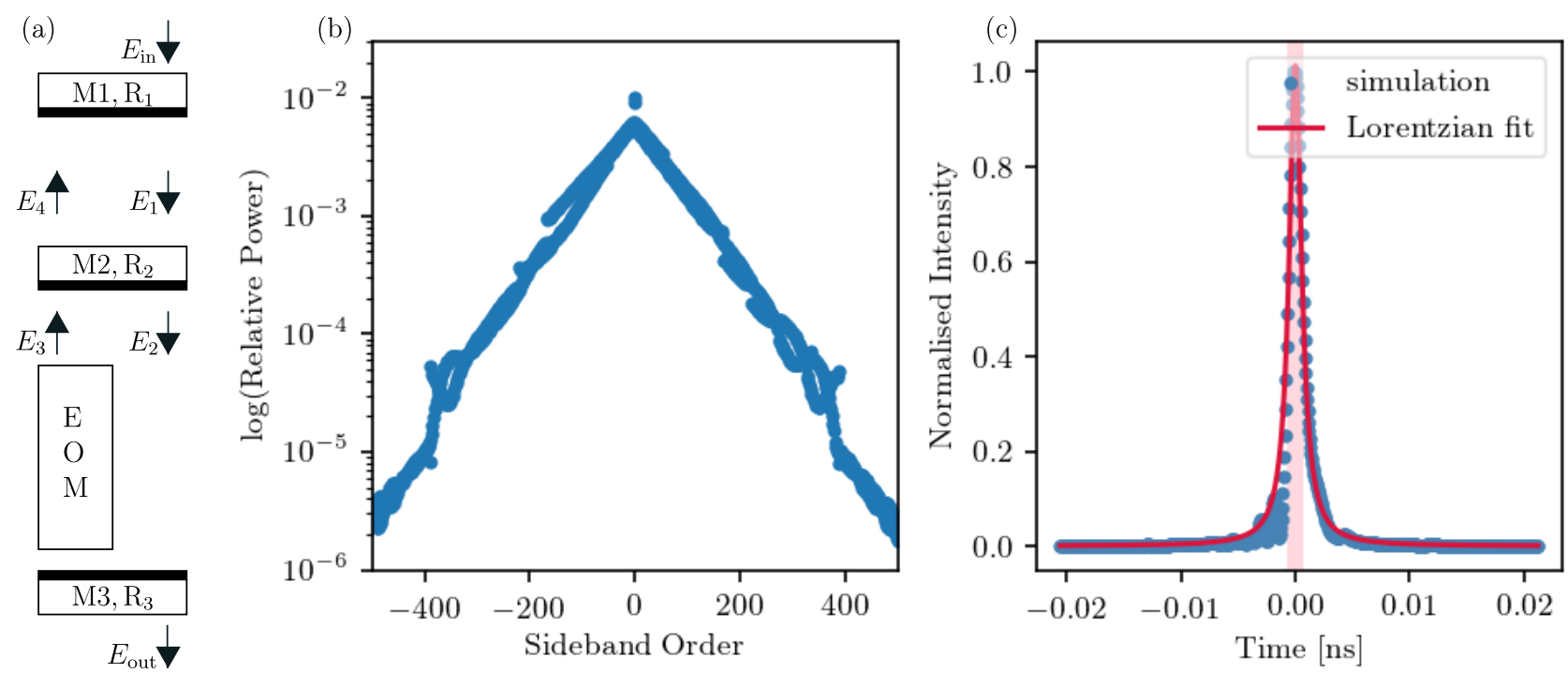

Figure 2: (a) Model of a coupled cavity OFCG. Again we are simplifying the maths by assuming the crystal is twice as long and light passes it only once per round-trip. (b) Spectral output of the coupled cavity OFCG. Incidental light beam is assumed to have a normalised intensity of 1 . Parameters used to obtain this plot were $R_{1}=R_{2}=0.99, R_{3}=0.96$, free spectral range of the coupling cavity was set to $21.13 \mathrm{GHz}$, while the second cavity was set to match the 5 th subharmonic of frequency of modulation $f_{m}=2.39 \mathrm{GHz}$. Modulation depth of the crystal $\delta=\pi / 2$, resulting in efficiency of the system of $90.4 \%$. (c) Temporal output from the coupled cavity OFCG with a FWHM pulse $\Delta \tau_{p}=1.33$ ps as indicated by the red area. Asymmetry is an artifact of the crystal dispersion and finite coupling matrix.

+ M3) containing the EOM, referred to as the EOM cavity. Here the coupling cavity is designed to be almost lossless, with impedance matched mirrors $R_{1}=R_{2}$ to allow for nearly $100 \%$ coupling into the EOM cavity. Using the mathematical model derived in Sec. A.2 we obtain the steady state of the system by solving the coupled equations

$$
\begin{gathered}
\vec{E}_{1}=\left[\mathbb{1}-\left(r_{1}^{\prime} t_{2}^{\prime} r_{3} t_{2} t_{\mathrm{c}}^{2} \hat{\phi}_{1} \times \hat{\phi}_{1} \times \hat{\phi}\left(\phi_{k}\right) \times M(2 \delta) \times\left\{\mathbb{1}-r_{2}^{\prime} r_{3} t_{\mathrm{c}}^{2} \hat{\phi}\left(\phi_{k}\right) \times M(2 \delta)\right\}^{-1}+r_{1}^{\prime} r_{2} \hat{\phi}_{1} \hat{\phi}_{1}\right)\right]^{-1} \times t_{1} \hat{\phi}_{1} \vec{E}_{\mathrm{in}}, \\
\vec{E}_{\text {out }}=t_{3} t_{\mathrm{c}} \hat{\phi}\left(\frac{\phi_{k}}{2}\right) \times M(\delta)\left[\mathbb{1}-r_{2}^{\prime} r_{3} t_{\mathrm{c}}^{2} \hat{\phi}\left(\phi_{k}\right) \times M(2 \delta)\right]^{-1} \times t_{2} \vec{E}_{1},
\end{gathered}
$$

where $t_{i}$ and $r_{i}$ are the transmitance and reflectivity of the mirror $M_{i}, \hat{\phi}_{1}$ is a phase change operator representing half round trip phase change between the sidebands in coupling cavity, $\hat{\phi}\left(\phi_{k}\right)$ is a phase operator representing full round trip phase change between the sidebands in the EOM cavity.

For the two cavity setup, an additional restriction on the frequency width of the generated comb arises when the $i^{\text {th }}$ sideband becomes resonant with the $j^{\text {th }}$ mode of the input coupling cavity. To maximise the comb-width, the FSR of the input cavity is chosen with a large minimum common denominator with the FSR of the EOM cavity, and a large finesse to reduce the cavity linewidth. The first sideband that is completely resonant with the coupling cavity is called the characteristic mode, which corresponds to the maxiumum sideband order of the comb.

To enhance photon pair generation using the source, the coupled cavity design was optimised to maximise the peak pulse energy whilst remaining below the damage threshold of the EOM. For the EOM crystal detailed in Sec. 2.1 to achieve $\delta=\pi / 2$ at a modulation frequency $f_{m}=2.39 \mathrm{GHz}$ the crystal aperture is limited to 

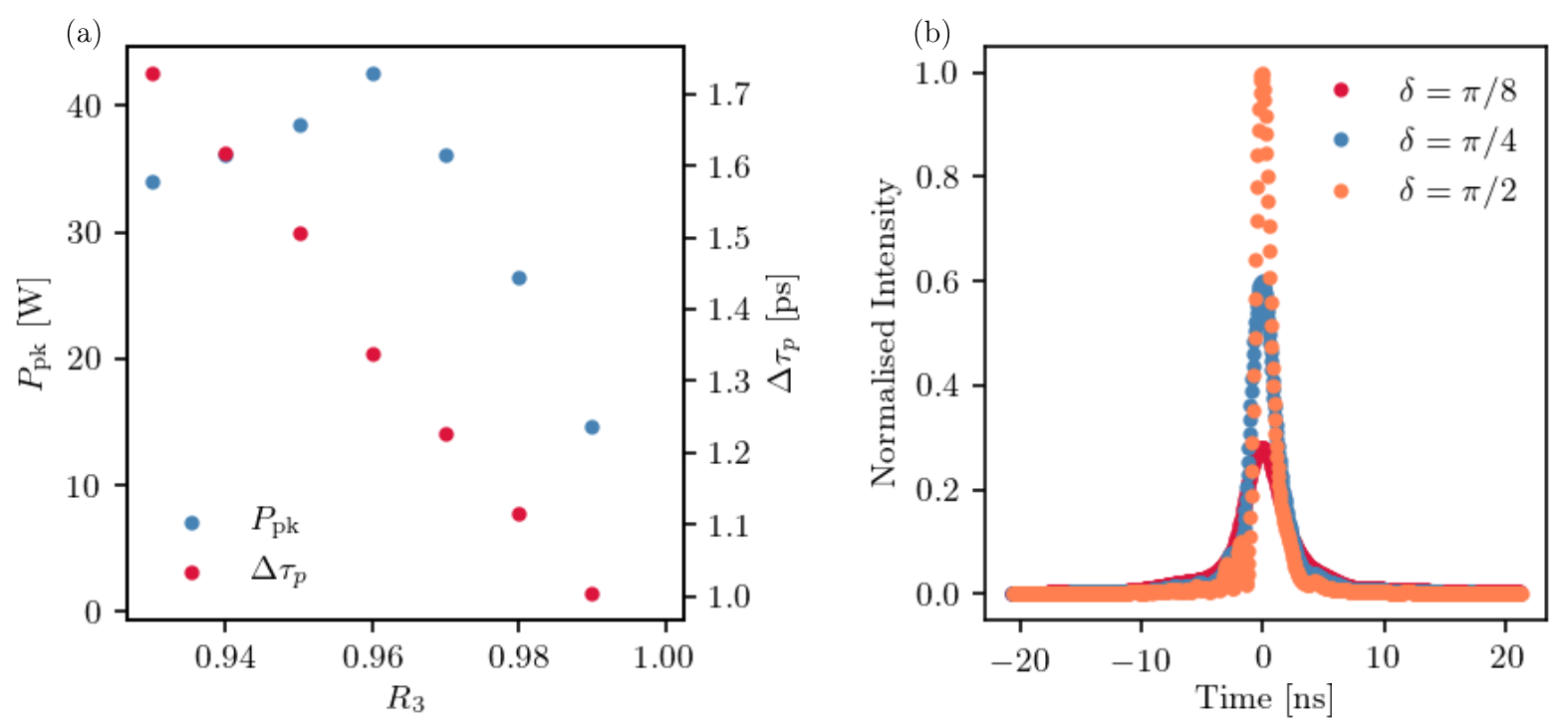

Figure 3: (a) Optimisation plot for the Coupled Cavity OFCG. Finesse of the EOM cavity was varied whilst $\delta=\pi / 2, f_{m}=2.39 \mathrm{GHz}, \mathrm{FSR}_{1}=21.13 \mathrm{GHz}, \mathrm{FSR}_{2}=478 \mathrm{MHz}$, and $R_{1}=R_{2}=0.99$ - the optimal value of the coupling cavity's finesse $(F=300)$. Additionally input power, was scaled so the intra-cavity intensity was below the damage threshold of the EOM crystal. On this plot the relation between peak power, pulse width and finesse can be observed. Increasing the finesse allows for shorter pulses but quickly ramps up the intra-cavity intensity, which places a restriction on $P_{\text {in }}$ and has negative impact on the peak power. (b) Variation of modulation depth while the system is operating with $f_{m}=2.39 \mathrm{GHz}$ and for optimised reflectivities of the mirrors $\left(R_{1}=R_{2}=\right.$ $\left.0.99 \& R_{3}=0.96\right)$. As we decrease the modulation depth below $\pi / 2$, the FWHM pulses $\Delta \tau_{p}$ become longer, respectively 1.3, 2.2 and $3.6 \mathrm{ps}$, which has negative effect on the peak power.

$2 \times 2 \mathrm{~mm}$, with a damage threshold of $20 \mathrm{~W} / \mathrm{mm}^{2}$ set by the AR coating. To mitigate this restriction the EOM cavity is extended to set $\mathrm{FSR}_{2}=f_{m} / 5$, equal to the $5^{\text {th }}$ subharmonic of the modulation frequency. The source performance is optimised through adjusting mirror reflectivities and radius of curvature to define beam waist in the EOM and the FSR of the input cavity. Possible values of FSR $_{1}$ were dictated by the curvatures of the mirrors allowing for desirable beam waist on the crystal facet. Final value of $\mathrm{FSR}_{1}=21.13 \mathrm{GHz}$ was chosen due to the characteristic mode appearing farthest away from the central mode in the spectrum. The peak power per pulse was calculated from

$$
P_{\mathrm{pk}}=\frac{\eta P_{\mathrm{in}}}{\Delta \tau_{p} 2 f_{m}},
$$

where $\eta$ is the efficiency of the EOM cycle, $P_{\text {in }}$ is the maximal average pump power of the system (up to 300 $\mathrm{mW}$ ) - limited by the damage threshold of the EOM crystal. Fig. 3(a) shows results of optimisation of the finesse of the EOM cavity. Fig. 3(b) shows the variation of the modulation depth in the system running with optimised parameters.

Following the optimisation the coupling cavity is chosen to have $\mathrm{FSR}_{1}=21.13 \mathrm{GHz}$ and $R_{1}=R_{2}=0.99$ yielding a finesse of 300 . The EOM cavity has $\mathrm{FSR}_{2}=478 \mathrm{MHz}$ leading to the characteristic mode of 1880 and finesse of 120. The simulated output field spectrum and temporal profile are shown in Fig. 2(b-c) at the optimised peak power, using system parameters $R_{1}=R_{2}=0.99, R_{3}=0.96, \delta=\pi / 2, f_{m}=2.39 \mathrm{GHz}$, FSR of the coupling cavity was $21.13 \mathrm{GHz}$, while the EOM cavity was set to match the $5^{\text {th }}$ subharmonic of frequency of modulation. For these parameters FWHM of the pulse width $\Delta \tau_{p}=1.33 \mathrm{ps}, \eta=90.4 \%$, the repetition rate equal to $4.78 \mathrm{GHz}$, peak power $42.4 \mathrm{~W}$, and beam waist $\omega_{0}=144 \mu \mathrm{m}$. 

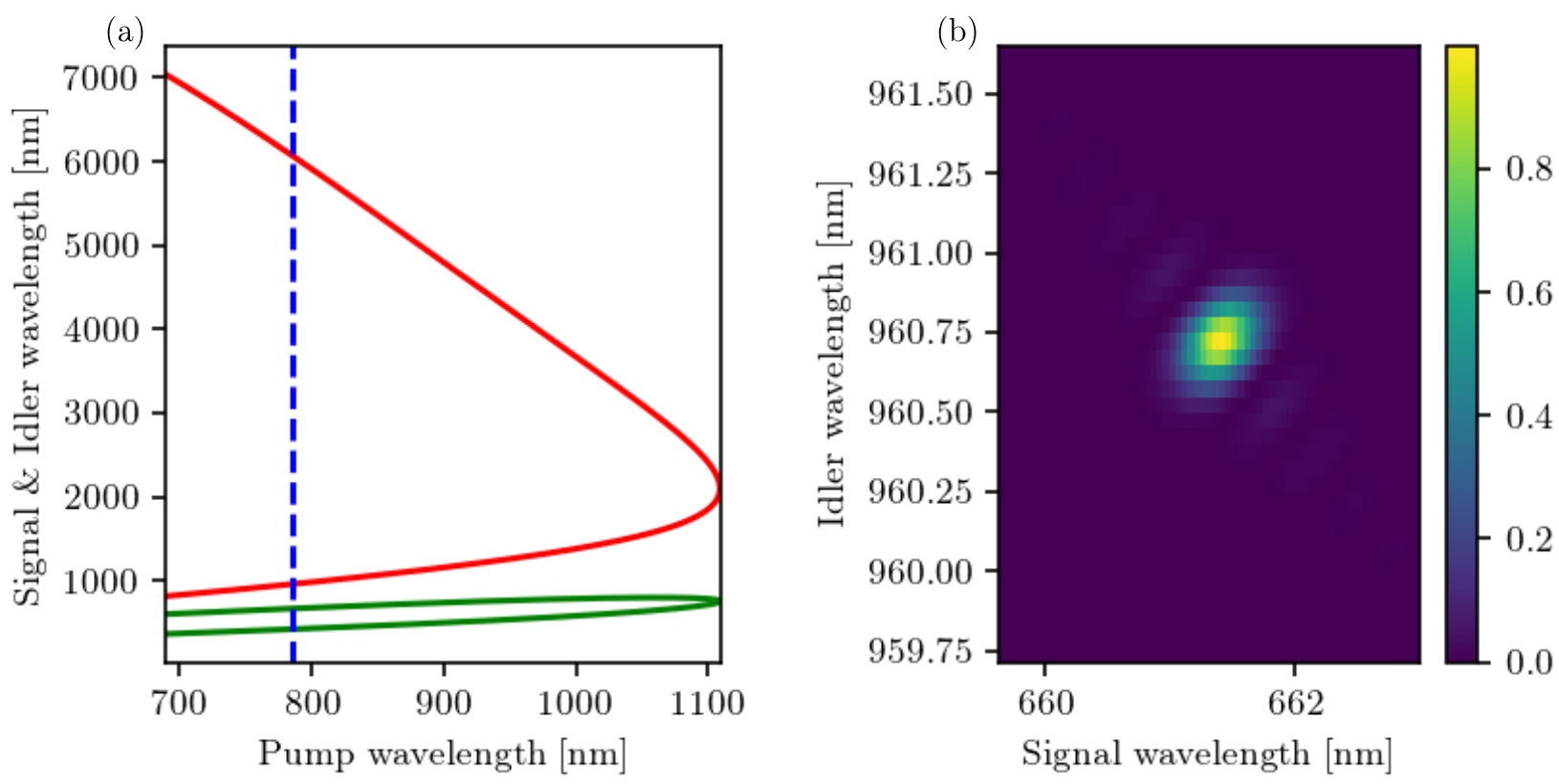

Figure 4: (a) Theoretical birefringent phase-matching contour as a function of the pump central wavelength $\lambda_{p}$. Signal wavelengths marked in green, idler wavelengths marked in red and blue dotted line marking the central frequency of the pump used. Values for the Fibercore HB800G optical fiber, operating in normal regime $\Delta n=4.3 \times 10^{-4}[15$ (b) Resulting joint spectral intensity of the generated signal \& idler pair, with purity of $71 \%$, fiber is assumed to be $L=10 \mathrm{~cm}$ long.

\section{PHOTON PAIR GENERATION}

For conversion of the output of the coupled cavity OFCG into spectrally pure and factorisable photon pairs for quantum illumination we propose use of FWM in a birefringent fiber, where photon pair frequencies are determined by energy conservation and by phase matching contour

$$
\kappa=k_{s}+k_{i}-2 k_{p}+\frac{2}{3} \gamma P_{p},
$$

where, $\kappa$ is the phase-mismatch between the propagation constants of the signal $(s)$, idler $(i)$ and two pump waves $(p)$ - caused by the chromatic dispersion in the fiber, with $k_{j}=\frac{n_{j} \omega_{j}}{c}(j=s, i, p)$ for light wave with angular frequency $\omega_{j}$ propagating in the medium with refractive index $n_{j}$.

For a HB800G fibre, a $785 \mathrm{~nm}$ pump wavelength is converted to a $\sim 660 \mathrm{~nm}$ and $\sim 960 \mathrm{~nm}$ signal and idler pair - shown in Fig. 4(a-b), ideally suited for demonstration of quantum LIDAR due to working with conventional GaAs SPAD detectors. The spectral purity of the created pair is determined by the joint spectral amplitude (JSA), $f\left(\omega_{s}, \omega_{i}\right) \stackrel{19}{19}$ In the ideal case of pure state generation the JSA of the output signal and idler photon pair should be separable - $f\left(\omega_{s}, \omega_{i}\right)=S\left(\omega_{s}\right) I\left(\omega_{i}\right)$. Here functions $S\left(\omega_{s}\right)$ and $I\left(\omega_{i}\right)$ represent the spectral amplitudes of the signal and idler fields.

To quantify the degree of inseparability (the degree of correlation between signal and idler modes) Schmidt decomposition of the JSA can be utilised. 28 The resulting Schmidt number $K$ represents the number of modes excited in FWM $(K=1)$ for a pure state, which is related to purity via $\mathcal{P}=1 / K{ }^{[28}$ For our system we consider a fiber length $10 \mathrm{~cm}$ corresponding to an estimated purity of $71 \%$ - shown on Fig. 4 (b), which can be increased using shorter fibers at the expense of reduced pair generation rates.

To estimate our expected pair detection rate we scale experimental data from, $\frac{15}{15}$ featuring a commercial pulsed Ti:Sapphire laser generating bandwidth limited pulses with $\Delta \lambda=3 \mathrm{~nm}$ centred at $704 \mathrm{~nm}$, with a repetition rate $R_{r}=80 \mathrm{MHz}$. For an $L_{c}=10 \mathrm{~cm}$ fiber, pair rates of $N_{\text {exp }}=23000$ pairs $/ \mathrm{s}$ are obtained from an average 
power of $15 \mathrm{~mW}$. Assuming sech ${ }^{2}$ pulse shape, this corresponds to pulse width of 0.17 ps with a peak pulse power of $P_{\mathrm{pk}, \text { ref }}=1.1 \mathrm{~kW}$. The pair production rate is linearly proportional to the fiber length $\mathrm{L}$ and quadratically proportional to peak power squared, $N \propto L P_{\mathrm{pk}}^{2}{ }^{29}$ This allows us to estimate the probability of creating a photon pair per pulse with $\alpha=N_{\exp } / R_{r}$, which then can be re-scaled using the data from Sec. 2.2 to obtain the predicted pair detection rate

$$
N=\left(\frac{P_{\mathrm{pk}}}{P_{\mathrm{pk}, \mathrm{ref}}}\right)^{2} \times \alpha \times 2 f_{m},
$$

where $P_{\mathrm{pk}}$ is peak power per pulse estimated from our model $(42.4 \mathrm{~W})$, and $2 f_{m}$ is the repetition rate of our system. We use the above to predict $>2000$ photon pairs detected per second.

\section{CONCLUSION}

We have presented a highly efficient coupled cavity OFCG optimised for use in generating spectrally pure photon pairs for use in quantum illumination experiments. The source provides a compact, low power and low cost approach to creating picosecond pulses with high $(\mathrm{GHz})$ repetition rate. We predict using photon pair generation in a commercial birefriengent single mode optical fiber to yield $>2000$ photon pairs/s, with photon pair purity of $71 \%$.

Unlike commercial pump sources, this design provides a dynamically tunable pulse width through controlling the EOM modulation depth. This method can be adapted to work at other wavelengths and in future extended to telecoms wavelengths. Exploration of the EOM materials offering higher damage thresholds will enable higher peak pulse energy and shorter pulse duration to boost the attainable pair generation rate. The purity of the photon pair generated can be increased by shortening the fibre to $L=6.81 \mathrm{~cm}$ to achieve highest pair purity attainable for this source of $80 \%$ at the cost of a reduction in photon pair production. Alternatively, a longer fiber can be chosen sacrificing purity for the increased pair generation rates.

\section{ACKNOWLEDGMENTS}

This project is funded by the UK Ministry of Defence.

\section{APPENDIX A. MATHEMATICAL MODELS}

\section{A.1 Single Cavity OFCG Mathematical model}

The output electric field can be calculated by considering the situation in Fig. 11(a). We are able to represent all of those electric fields as follows

$$
\begin{aligned}
& \vec{E}_{1}=t_{1} \vec{E}_{\text {in }}+r_{1} \vec{E}_{2}, \\
& \vec{E}_{2}=t_{\mathrm{c}}^{2} \hat{\phi}\left(\phi_{k}\right) M(2 \delta) \times \vec{E}_{1},
\end{aligned}
$$

where $r_{i}$ and $t_{i}$ stand for reflectivity and transmittance of mirror $\mathrm{M}_{i}$ and $t_{\mathrm{c}}$ is transmittance trough the cavity. Operator $\hat{\phi}\left(\phi_{k}\right)$ is the full round-trip phase change due to crystal dispersion and can be expressed as

$$
\hat{\phi}\left(\phi_{k}\right)=\left[\begin{array}{ccccc}
e^{-i \phi_{k}} & 0 & \cdots & \cdots & 0 \\
0 & \ddots & \ddots & & \vdots \\
\vdots & \ddots & e^{-i \phi_{0}} & \ddots & \vdots \\
\vdots & & \ddots & \ddots & 0 \\
0 & \cdots & \cdots & 0 & e^{-i \phi_{-k}}
\end{array}\right]
$$


where $k$ is the sideband order and $\phi_{k}$ is the phase change due to crystal dispersion per sideband, given by Eq. (4). $M(2 \delta)$ is the transformation matrix of the EOM. This matrix can be represented as

$$
M(2 \delta)=\left[\begin{array}{ccccc}
J_{0}(2 \delta) & -J_{1}(2 \delta) & J_{2}(2 \delta) & \ldots & J_{2 k}(2 \delta) \\
J_{1}(2 \delta) & J_{0}(2 \delta) & -J_{1}(2 \delta) & \ldots & -J_{2 k-1}(2 \delta) \\
J_{2}(2 \delta) & J_{1}(2 \delta) & J_{0}(2 \delta) & \ldots & J_{2 k-2}(2 \delta) \\
\vdots & \vdots & \vdots & \ddots & \vdots \\
J_{2 k}(2 \delta) & J_{2 k-1}(2 \delta) & J_{2 k-2}(2 \delta) & \ldots & J_{0}(2 \delta)
\end{array}\right]
$$

where $J_{k}(\delta)$ is Bessel function identity of order $i$, and an origin $\delta$, and where $\delta$ is the modulation depth.

Using Eq. 10a - 10b we can solve the field state in $\vec{E}_{1}$ depending on incident field $\vec{E}_{\text {in }}$

$$
\vec{E}_{1}=\left[\mathbb{1}-r_{1} r_{2} t_{\mathrm{c}}^{2} \hat{\phi}\left(\phi_{k}\right) \times M(2 \delta)\right]^{-1} \times t_{1} \vec{E}_{\mathrm{in}},
$$

using this solution, we can calculate output electric field of the OFCG - $\vec{E}_{\text {out }}$, to be

$$
\vec{E}_{\text {out }}=t_{\mathrm{c}} \hat{\phi}\left(\frac{\phi_{k}}{2}\right) \times M(\delta) \times\left(t_{2} \vec{E}_{1}\right) .
$$

\section{A.2 Coupled Cavity OFCG Mathematical Model}

To solve the coupled cavity OFCG we take situation in Fig. 2(a)

$$
\begin{aligned}
& \vec{E}_{1}=\hat{\phi}_{1} \times\left(t_{1} \vec{E}_{\mathrm{in}}+r_{1}^{\prime} \vec{E}_{4}\right), \\
& \vec{E}_{2}=t_{2} \vec{E}_{1}+r_{2}^{\prime} \vec{E}_{3}, \\
& \vec{E}_{3}=t_{\mathrm{c}}^{2} \hat{\phi}_{2} \times M(2 \delta) \times r_{3} \vec{E}_{2}, \\
& \vec{E}_{4}=\hat{\phi}_{1} \times\left(t_{2}^{\prime} \vec{E}_{3}+r_{2} \vec{E}_{1}\right),
\end{aligned}
$$

where $\hat{\phi}_{1}$ is a phase operator, representing half round trip phase change between sidebands, in first cavity, $\hat{\phi}_{2}$ is a phase operator, representing full round trip phase change between sidebands in the EOM cavity, $\mathrm{M}(2 \delta)$ is full round trip transformation matrix, $r(t)_{i}$ represent mirror $\mathrm{M}_{i}$ reflectivity (transmitance) on left side of mirrors respectively, while $r^{\prime}\left(t^{\prime}\right)_{i}$ represent mirror $\mathrm{M}_{i}$ reflectivity (transmitance) on the right side of the mirrors. relation between those can be represented as

$$
\begin{aligned}
r^{\prime} & =r, \\
t^{\prime} & =-t,
\end{aligned}
$$

this ensures the transmission phase is preserved. By substituting Eq. (15c) into Eq. (15b) we obtain

$$
\begin{aligned}
& \vec{E}_{2}=t_{2} \vec{E}_{1}+r_{2}^{\prime} r_{3} t_{\mathrm{c}}^{2}\left(\hat{\phi}_{2} \times M(2 \delta) \times \vec{E}_{2}\right), \\
& \vec{E}_{2}=\left[\mathbb{1}-r_{2}^{\prime} r_{3} t_{\mathrm{c}}^{2} \hat{\phi}_{2} \times M(2 \delta)\right]^{-1} \times t_{2} \vec{E}_{1},
\end{aligned}
$$

after that we substitute Eq. [15c into Eq. (15d) to obtain

$$
\vec{E}_{4}=\hat{\phi}_{1}\left(t_{2}^{\prime} r_{3} t_{2} t_{\mathrm{c}}^{2} \hat{\phi}_{2} \times M(2 \delta)\left[\mathbb{1}-r_{2}^{\prime} r_{3} t_{\mathrm{c}}^{2} \hat{\phi}_{2} M(2 \delta)\right]^{-1} \times \vec{E}_{1}+r_{2} \vec{E}_{1}\right)
$$

finally we substitute Eq. $17 \mathrm{~b}$ and Eq. 18 into $15 \mathrm{a}$, to solve the steady state of the system

$$
\vec{E}_{1}=\left[\mathbb{1}-\left(r_{1}^{\prime} t_{2}^{\prime} r_{3} t_{2} t_{\mathrm{c}}^{2} \hat{\phi}_{1} \times \hat{\phi}_{1} \times \hat{\phi}_{2} \times M(2 \delta) \times\left\{\mathbb{1}-r_{2}^{\prime} r_{3} t_{\mathrm{c}}^{2} \hat{\phi}_{2} \times M(2 \delta)\right\}^{-1}+r_{1}^{\prime} r_{2} \hat{\phi}_{1} \hat{\phi}_{1}\right)\right]^{-1} \times t_{1} \hat{\phi}_{1} \vec{E}_{\mathrm{in}},
$$


now to obtain the output electric field we substitute Eq. (19) into Eq. [17b), and multiply the result by transmittance of the third mirror, half round trip phase operator in the EOM cavity, and half round trip transformation matrix.

$$
\vec{E}_{\text {out }}=t_{3} t_{\mathrm{c}} \hat{\phi}\left(\frac{\phi_{k}}{2}\right) \times M(\delta) \vec{E}_{2}
$$

\section{REFERENCES}

[1] Kok, P., Munro, W. J., Nemoto, K., Ralph, T. C., Dowling, J. P., and Milburn, G. J., "Linear optical quantum computing with photonic qubits", Rev. Mod. Phys. 79, 135-174 (2007).

[2] Loredo, J. C., Broome, M. A., Hilaire, P., Gazzano, O., Sagnes, I., Lemaitre, A., Almeida, M. P., Senellart, P., and White, A. G., "Boson sampling with single-photon fock states from a bright solid-state source", Phys. Rev. Lett. 118, 130503 (2017).

[3] Harris, S. E., "Chirp and compress: Toward single-cycle biphotons", Phys. Rev. Lett. 98 (2007).

[4] V. Giovannetti, S. L. and Maccone, L., "Quantum-enchanced positioning and clock synchronization", $N a$ ture 412, 417-419 (2001).

[5] Franson, J. D., "Nonclassical nature of dispersion cancellation and nonlocal interferometry", Phys. Rev. A 80 (2009).

[6] England, D. G., Balaji, B., and Sussman, B. J., "Quantum-enhanced standoff detection using correlated photon pairs", Phys. Rev. A 99 (Feb 2019).

[7] Lloyd, S., "Enhanced sensitivity of photodetection via quantum illumination", Science 321, 1463-1465 (2008).

[8] Tan, S.-H., Erkmen, B. I., Giovannetti, V., Guha, S., Lloyd, S., Maccone, L., Pirandola, S., and Shapiro, J. H., "Quantum illumination with gaussian states", Phys. Rev. Lett. 101, 253601 (2008).

[9] Shapiro, J. H. and Lloyd, S., "Quantum illumination versus coherent-state target detection", New J. Phys. 11, 063045 (2009).

[10] Guha, S. and Erkmen, B. I., "Gaussian-state quantum-illumination receivers for target detection", Phys. Rev. A 80, 052310 (2009).

[11] Zhang, Z., Mouradian, S., Wong, F. N., and Shapiro, J. H., "Entanglement-enhanced sensing in a lossy and noisy environment", Phys. Rev. Lett. 114 (2015).

[12] Frick, S., McMillan, A., and Rarity, J., "Quantum rangefinding", Opt. Express 28, 37118 (2020).

[13] Yang, H., Roga, W., Pritchard, J. D., and Jeffers, J., "Gaussian state-based quantum illumination with simple photodetection", Opt. Express 29, 8199 (2021).

[14] Kaneda, F., Garay-Palmett, K., U'Ren, A. B., and Kwiat, P. G., "Heralded single-photon source utilizing highly nondegenerate, spectrally factorable spontaneous parametric downconversion", Opt. Express 24, 10733-10747 (2016).

[15] Smith, B. J., Mahou, P., Cohen, O., Lundeen, J. S., and Walmsley, I. A., "Photon pair generation in birefringent optical fibers", Opt. Express 17, 23589-23602 (2009).

[16] Clark, A. S., Fulconis, J., Rarity, J. G., Wadsworth, W. J., and O’Brien, J. L., “All-optical-fiber polarizationbased quantum logic gate", Phys. Rev. A 79, 030303 (Mar 2009).

[17] Matthews, J. C. F., Politi, A., Stefanov, A., and O'Brien, J. L., "Manipulating multi-photon entanglement in waveguide quantum circuits", Nat. Photonics 3 (2009).

[18] Smith, B. J., Kundys, D., Thomas-Peter, N., Smith, P. G. R., and Walmsley, I. A., "Phase-controlled integrated photonic quantum circuits", Opt. Express 17 (2009).

[19] Lugani, J., Francis-Jones, R. J. A., Boutari, J., and Walmsley, I. A., "Spectrally pure single photons at telecommunications wavelengths using commercial birefringent optical fiber", Opt. Express 28, 5147 (2020).

[20] Kovacich, R. P., Sterr, U., and Telle, H. R., "Short-pulse properties of optical frequency comb generators", Appl. Opt. 39, 4372-4376 (2000).

[21] Kourogi, M., Nakagawa, K., and Ohtsu, M., "Wide-span optical frequency comb generator for accurate optical frequency difference measurement", IEEE J. Quantum Electron. 29, 2693-2701 (1993). 
[22] Kobayashi, T., Sueta, T., Cho, Y., and Matsuo, Y., "High-repetition-rate optical pulse generator using a fabry-perot electro-optic modulator", Appl. Phys. Lett. 21, 341-343 (1972).

[23] Macfarlane, G. M., Bell, A. S., Riis, E., and Ferguson, A. I., "Optical comb generator as an efficient short-pulse source", Opt. Lett. 21, 534-536 (1996).

[24] Kourogi, M., Widiyatomoko, B., Takeuchi, Y., and Ohtsu, M., "Limit of optical-frequency comb generation due to material dispersion", IEEE J. Quantum Electron. 31, 2120-2126 (1995).

[25] Xiao, S., Hollberg, L., Newbury, N. R., and Diddams, S. A., "Toward a low-jitter 10 ghz pulsed source with an optical frequency comb generator", Opt. Express 16 (2008).

[26] Moutzouris, K., Hloupis, G., Stavrakas, I., Triantis, D., and Chou, M.-H., "Temperature-dependent visible to near-infrared optical properties of $8 \mathrm{~mol} \%$ mg-doped lithium tantalate", Opt. Mater. Express 1, 458-465 (2011).

[27] Xiao, S., Hollberg, L., and Diddams, S. A., "Generation of a 20 ghz train of subpicosecond pulses with a stabilized optical-frequency-comb generator", Opt. Lett. 34, 85-87 (2009).

[28] Zielnicki, K., Garay-Palmett, K., Cruz-Delgado, D., Cruz-Ramirez, H., O’Boyle, M. F., Fang, B., Lorenz, V. O., U'Ren, A. B., and Kwiat, P. G., "Joint spectral characterization of photon-pair sources", J. Mod. Opt. 65, 1141-1160 (2018).

[29] Lin, Q., Yaman, F., and Agrawal, G. P., "Photon-pair generation in optical fibers through four-wave mixing: Role of raman scattering and pump polarization", Phys. Rev. A 75, 023803 (2007). 